\title{
Ultrastructural Analysis of Cytoskeletal Networks in Neuronal Growth Cones by Light and Electron Microscopy
}

Ryan Hylton ${ }^{1}$, Michael Grillo ${ }^{2}$, Jessica Heebner ${ }^{2}$ and Matthew Swulius ${ }^{2}$

${ }^{1}$ Penn State College of Medicine, Hershey, Pennsylvania, United States, ${ }^{2}$ Penn State College of Medicine, United States

In the brain, proper neural circuits are built by the directed growth of nascent axons and dendrites towards their eventual synaptic partners through a process termed neurite guidance. At the distal tip of these neurites exists a subcellular compartment termed the growth cone. The growth cone integrates environmental cues, both chemical and mechanical, and converts them into mechanical force to move the developing neurite towards its target ${ }^{1-3}$.

Translocation of the growth cone is largely facilitated by the combined polymerization and depolymerization of actin filaments (F-actin) in its peripheral (or "P") domain. The P-domain contains two actin-based structures: filopodia and lamellipodia. Filopodia are finger-like protrusions shaped by tightly bundled, unipolar actin filaments and serve as antennae for the growth cone, finding and responding to extracellular cues ${ }^{3}$. Lamellipodia consist of networks of branched actin filaments between filopodia at the leading edge of the cell. Actin polymerization here propels the plasma membrane, and the growth cone itself, forward ${ }^{3}$.

During neurite guidance, the growth cone is steered towards its destination by the collective response to both attractive and repulsive cues ${ }^{1}$. In general, attractive cues result in signaling cascades that facilitate actin polymerization and forward movement in the direction of the incoming signal. Conversely, repulsive cues lead to depolymerization of actin networks. The synchronized action of both responses leads to growth in the direction of the attractive cue.

In this way, a delicate balance of actin dynamics must be maintained for proper growth cone motility. Factin possesses the ability to grow, shrink, sever, branch, and bundle when assisted by the right actin binding proteins ${ }^{4}$. In fact, a number of these proteins have been shown to be crucial for growth cone outgrowth and/or guidance ${ }^{5-8}$. Here, we focus predominantly on two of these proteins. First, fascin is a crosslinking protein whose primary function is the bundling of F-actin in filopodia of motile cells, including the growth cone ${ }^{9,10}$. Second, cofilin binds to old, ADP-bound actin filaments and facilitates their severing and depolymerization ${ }^{11,12}$. We have investigated the impact these two proteins have on growth cone cytoskeletal architecture, and will present these findings here.

Cytoskeletal remodeling in growth cones has long been studied by fluorescence light microscopy. These papers revealed a wealth of information regarding growth cone morphology, signaling pathways, and overall mechanisms of translocation ${ }^{1-3}$. However, much less investigation has been done on the detailed ultrastructure of the growth cone cytoskeleton. Here, we examine the structural role fascin and cofilin play during growth cone outgrowth by both light and electron microcopy. We use immunofluorescence on fixed cells to guide our understanding of the localization of these proteins within the growth cone. We also use live cell fluorescence microscopy to investigate their effect on growth cone filopodial dynamics. Finally, we utilize cryo-electron tomography and, subsequently, subtomogram averaging and Al-based tomographic segmentation to investigate the molecular structure of actin filaments and the ultrastructure of filament networks, respectively. 

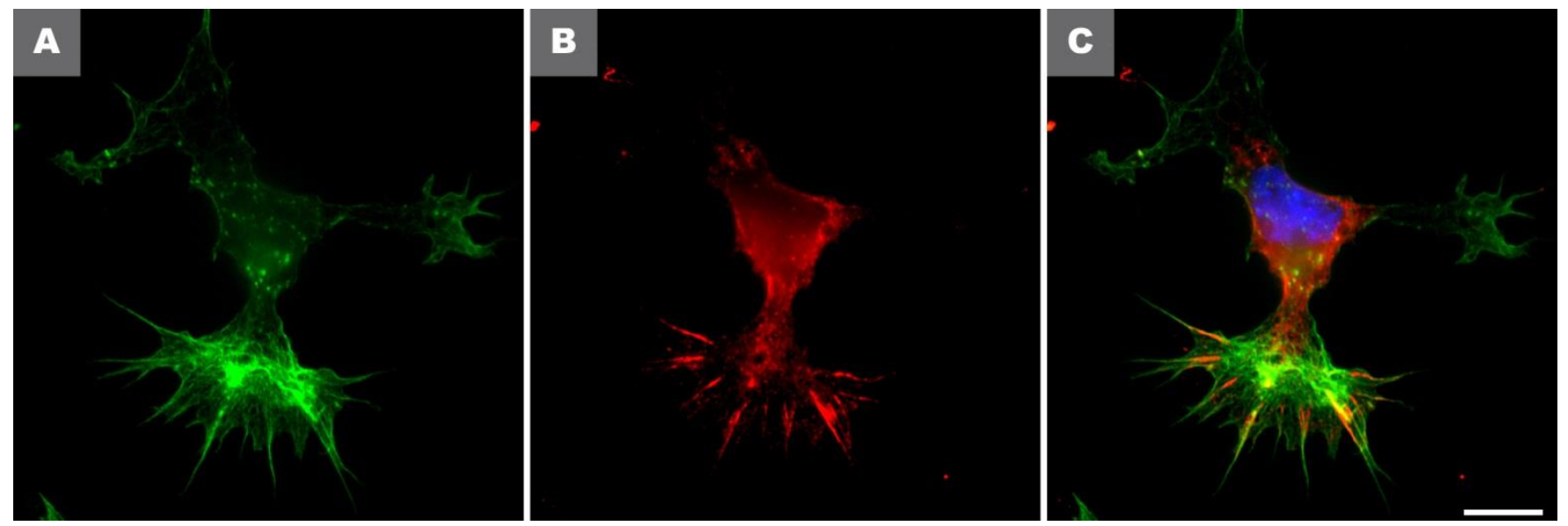

Figure 1. Fluorescence microscopy of neuronal growth cones. Immunofluorescence of a hippocampal neuron on DIV 1 shows that cofilin (red) primarily localizes near the base of growth cone filopodia in linear aggregates. A) Phalloidin/actin labeled with Alexa Fluor 488. B) Cofilin labeled with Alexa Fluor 594. C) Merged image of the channels shown in A) and B), along with DAPI in blue. Scale bar: $10 \mu \mathrm{m}$.
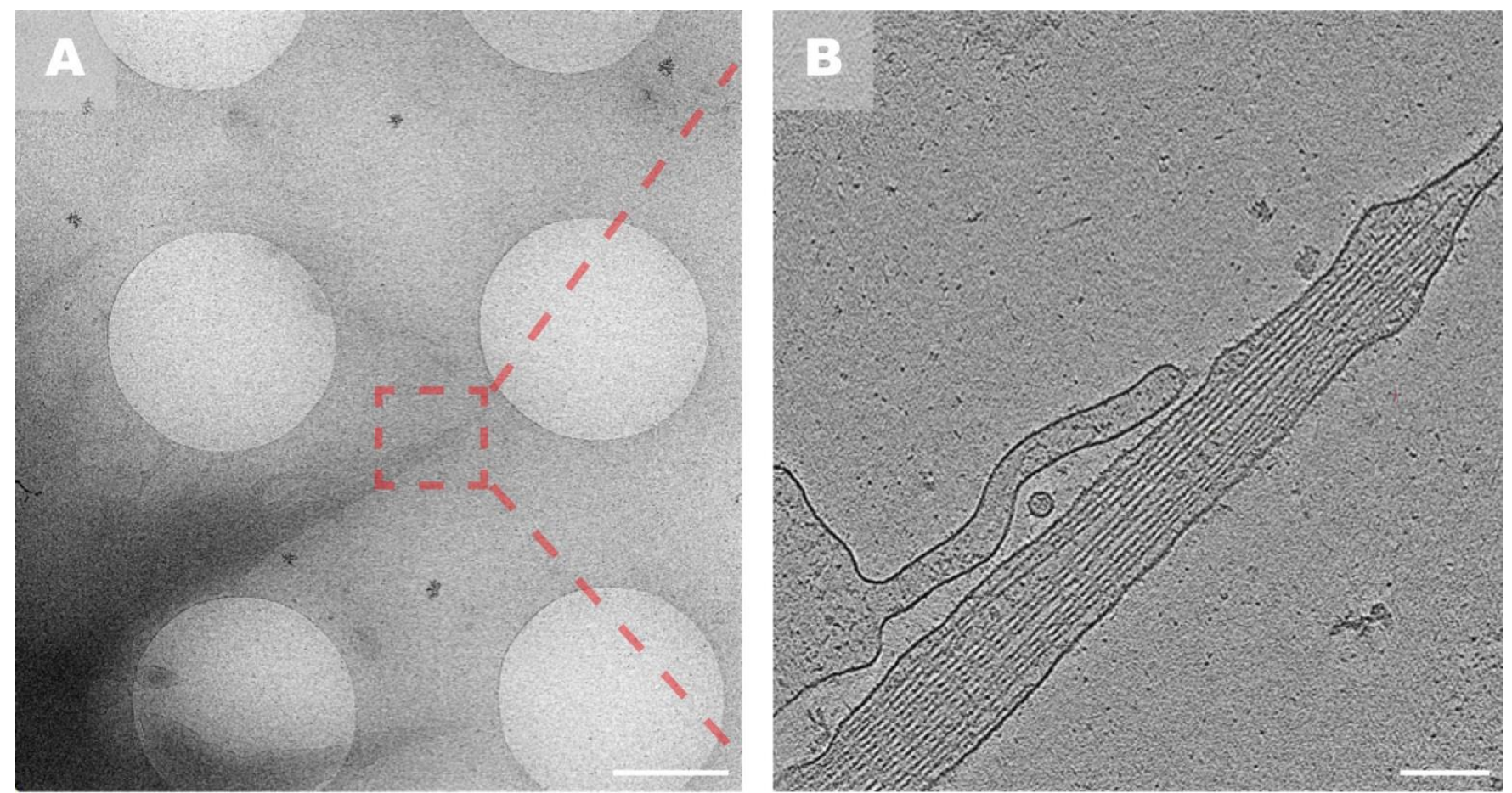

Figure 2. Cryo-electron tomography of neuronal growth cones. A) TEM overview image showing a growth cone on a Quantifoil EM grid. Dashed box shows the location of the tomogram in B). B) $8.6 \mathrm{~nm}$ thick slice of a tomogram showing a growth cone filopodium. A bundle of actin filaments can be seen surrounded by the plasma membrane. Scale bars: A) $1 \mu \mathrm{m}$ and B) $150 \mathrm{~nm}$.

\section{References}

1. Vitriol E, Zheng J. Growth Cone Travel in Space and Time: The Cellular Ensemble of Cytoskeleton, Adhesion, and Membrane. Neuron (Cambridge, Mass.). 2012;73:1068-1081.

2. Kerstein PC, Nichol RH, Gomez TM. Mechanochemical regulation of growth cone motility. Frontiers in cellular neuroscience. 2015;9:244-244. 
3. Lowery LA, Vactor DV. The trip of the tip: understanding the growth cone machinery. Nature reviews. Molecular cell biology. 2009;10:332-343.

4. Pollard TD. Actin and actin-binding proteins. Cold Spring Harbor perspectives in biology. 2016;8:a018226.

5. Flynn KC, Bamburg JR, Pak CW. Actin-binding proteins take the reins in growth cones. Nature reviews. Neuroscience. 2008;9:136-147.

6. Dent EW, Gupton SL, Gertler FB. The growth cone cytoskeleton in axon outgrowth and guidance. Cold Spring Harbor perspectives in biology. 2011;3:1-39.

7. De Arcangelis A, Georges-Labouesse E, Adams JC. Expression of fascin-1, the gene encoding the actin-bundling protein fascin-1, during mouse embryogenesis. Gene Expression Patterns. 2004;4:637643.

8. Marsick BM, Flynn KC, Santiago-Medina M, Bamburg JR, Letourneau PC. Activation of ADF/cofilin mediates attractive growth cone turning toward nerve growth factor and netrin-1. Developmental neurobiology (Hoboken, N.J.). 2010;70:565-588.

9. Jayo A, Parsons M. Fascin: A key regulator of cytoskeletal dynamics. The international journal of biochemistry \& cell biology. 2010;42:1614-1617.

10. Cohan CS, Welnhofer EA, Zhao L, Matsumura F, Yamashiro S. Role of the actin bundling protein fascin in growth cone morphogenesis: Localization in filopodia and lamellipodia. Cell motility and the cytoskeleton. 2001;48:109-120.

11. Kanellos G, Frame MC. Cellular functions of the ADF/cofilin family at a glance. Journal of cell science. 2016;129:3211-3218.

12. Gungabissoon RA, Bamburg JR. Regulation of Growth Cone Actin Dynamics by ADF/Cofilin. The journal of histochemistry and cytochemistry. 2003;51:411-420. 